\title{
Alinhamento estratégico de melhoria de processos de software: percepções de um processo de apoio à decisão
}

\author{
Francisco J. S. Vasconcellos ${ }^{1}$, Caíque Minhare ${ }^{1}$, Leonardo Fuchs ${ }^{1}$, \\ Jucele F. A. Vasconcellos ${ }^{1}$, José Adson O. G. da Cunha ${ }^{2}$, Auri M.R. Vincenzi ${ }^{3}$ \\ ${ }^{1}$ Faculdade de Computação, Universidade Federal de Mato Grosso do Sul \\ Caixa Postal 549 - 79.070-900 - Campo Grande, MS - Brasil \\ ${ }^{2}$ Departamento de Ciências Exatas - Universidade Federal da Paraíba \\ Rio Tinto, PB - Brasil \\ ${ }^{3}$ Departamento de Ciência da Computação -Universidade Federal de São Carlos \\ São Carlos, SP - Brasil \\ \{francisco.vasconcellos, jucele\} @facom.ufms.br,
\{caique.minhare, leonardo.fuchs\}@aluno.ufms.br,
adson@dcx.ufpb.br, auri@dc.ufscar.br
}

\begin{abstract}
The alignment between software process improvement (SPI) and organizational goals involves strategic and tactical decision making. This article presents a qualitative study about the usefulness of a formal decisionmaking process in the strategic alignment of SPI. The definition and refinement of the proposed process helped to develop theoretical propositions in an inductive way. Questionnaires adapted from the Technology Acceptance Model positively evaluated both the ease of use and the perceived utility of the proposed solution. The results indicate that a formal decision-making process is useful and necessary for the strategic alignment of SPI.
\end{abstract}

Resumo. O alinhamento de melhoria do processo de software (MPS) com objetivos organizacionais envolve decisões em níveis estratégico e tático. Este artigo apresenta um estudo qualitativo sobre a utilidade de um processo formal de tomada de decisão no alinhamento estratégico de MPS. A definição e o refinamento do processo proposto ajudaram a desenvolver proposições teóricas de forma indutiva. Questionários adaptados do Modelo de Aceitação de Tecnologia avaliaram positivamente tanto a facilidade de uso quanto a utilidade percebida da solução proposta. Os resultados indicam que um processo formal de tomada de decisão é útil e necessário para o alinhamento estratégico de MPS.

\section{Introdução}

A melhoria de processos de software (MPS) tem como objetivo aumentar a eficácia de uma organização de software e a seleção das práticas a serem melhoradas depende do contexto organizacional, cultura e prioridades (Humphrey 1989). Ebert (1999) afirma que os objetivos estratégicos organizacionais devem estar claros antes da definição de metas de melhoria pelo nível tático. De fato, estudos indicam o alinhamento com os objetivos de negócios como um fator crítico para o sucesso de MPS (Dybå 2005). Além disso, a norma ISO/IEC 33014 enfatiza que, quanto maior o alinhamento entre objetivos de negócios e as decisões de melhoria de processos, maior a probabilidade de sucesso do programa de melhorias (ISO/IEC 2013). 
Motivados pelo estudo de Lepmets et al. (2012), Vasconcellos et al. (2017) avaliam, em uma revisão sistemática da literatura (RSL), 19 abordagens para o alinhamento estratégico de MPS. Entre outros aspectos, a falta de validação empírica indica uma potencial falha na transferência adequada das soluções para a prática. Além disso, nenhum método foi sugerido para apoiar o processo de tomada de decisão em um alinhamento estratégico de MPS. Considerando a tomada de decisões como habilidade que, como tal, pode ser aprimorada com experiência e treinamento, entende-se que esta também pode ser auxiliada por processos que forneçam mecanismos de apoio à decisão (Hastie e Dawes 2010). Assim, é importante considerar o emprego de métodos de apoio à decisão e de Sistemas de Apoio à Decisão (SAD), para ajudar os profissionais a decidirem sobre melhorias nos processos de software. Dentre os 51 estudos avaliados na RSL, apenas um trabalho apresenta uma técnica para apoio à decisão, mas não a emprega de modo a alinhar a seleção de melhorias no processo a objetivos organizacionais. Outros estudos apresentam abordagens para a seleção e para o monitoramento de ações de melhoria alinhadas a objetivos organizacionais, mas com baixa evidência de efetiva transferência para a prática, o que nos remete à seguinte questão de pesquisa:

Como organizações de software percebem a utilidade de um Sistema de Apoio à Decisão para prover e manter o alinhamento entre ações de melhoria de processos de software e suas necessidades organizacionais?

O objetivo deste trabalho relaciona-se ao desenvolvimento do conhecimento sobre a utilidade percebida, pelos profissionais, de um sistema de apoio à tomada de decisões, representado por um processo e ferramental de apoio, aplicado ao alinhamento estratégico de MPS. Este artigo apresenta resultados de duas fases de validação, de um método composto por três fases, que busca a transferência adequada de uma solução proposta para a indústria. Inspirado nos trabalhos de Shull et al. (2001) e pelo modelo de transferência de tecnologia proposto por Gorschek et al. (2006), foram delineados um processo e uma ferramenta de apoio, inicialmente com base no referencial teórico (seção 2). Partindo da premissa de que um processo formal de tomada de decisões pode ser útil para o alinhamento entre os objetivos da alta administração com as escolhas de melhoria do processo, conjecturas iniciais foram aprimoradas por indução analítica (Johnson 2004) através de um método iterativo de validação (seção 3). Os resultados contribuíram para o aperfeiçoamento do conhecimento e para o refinamento da solução, possibilitando assim, a análise apresentada na seção 4. Considerando as ameaças à validade (seção 5), as conclusões e trabalhos futuros são apresentados na seção 6.

\section{Referencial Teórico}

A proposição teórica inicial, composta por conjecturas, considera alguns constructos e relações apoiadas pela revisão da literatura, como recomendam métodos qualitativos de construção de teorias (Merriam 2009; Corbin e Strauss 2015). Além dos conceitos necessários para as observações iniciais e para o estímulo de questões utilizadas durante o processo de pesquisa, a literatura também contribuiu para aprimorar a sensibilidade teórica do segundo e terceiro autores, iniciantes nesta área substantiva (Corbin e Strauss, 2015). Nesta seção são descritos conceitos relacionados ao alinhamento estratégico de MPS e ao processo de tomada de decisão correspondente. As conjecturas iniciais são listadas com identificadores de $\mathrm{C}_{1}$ a $\mathrm{C}_{7}$. 


\subsection{Alinhamento estratégico de MPS}

Organizações de software com ou sem fins lucrativos possuem metas organizacionais definidas pela alta administração. As melhorias em um processo de software podem ser derivadas de metas organizacionais em um planejamento de MPS top-down (ISO/IEC 2013) ou alinhadas com os objetivos de negócios em uma estratégia de MPS bottom-up, comum em um ambiente ágil (Lepmets e McBride 2012).

Independentemente do cenário, uma iniciativa de MPS invariavelmente exige mudanças em um processo de software. Münch et al. (2012) afirmam que mudanças nos processos de software devem ser cuidadosamente planejadas porque, para serem eficazes, dependem dos seres humanos, do contexto organizacional e do ambiente de negócios, representando assim, custos significativos para uma organização. Qualquer mudança em um processo de software pode então ser vista como uma decisão estratégica. Como o alinhamento de MPS envolve tais decisões estratégicas, este será denominado alinhamento estratégico de MPS.

O modelo COBIT (ISACA 2012) e o padrão ISO/IEC (ISO/IEC 2015) estabelecem três tarefas principais relacionadas à governança de tecnologia da informação (TI): dirigir, monitorar e avaliar. Além disso, o padrão ISO/IEC recomenda a delegação de responsabilidades específicas a gerentes funcionais de TI (o gerente de esforços de melhoria de processos no contexto deste estudo). Assim, são apresentadas duas conjecturas iniciais:

C1: O alinhamento estratégico de MPS envolve o planejamento e o monitoramento de atividades, demandando tanto sistemas para apoio à tomada de decisão quanto para a mensuração de resultados.

C2: $O$ alinhamento estratégico de MPS envolve duas partes interessadas principais: diretores e gerentes de MPS.

Trienekens et al. (2005) enfatizam o papel do processo de tomada de decisão na decomposição da estratégia e metas de negócios em metas de equipes técnicas. Como mostrado na Figura 1, são três níveis de tomada de decisão: estratégico, tático e operacional. A decisão estratégica refere-se à priorização de objetivos de negócios (o "porquê"). Gerentes de MPS tomam decisões táticas, selecionando alternativas de melhorias (o "que") alinhadas com as decisões estratégicas. A decisão operacional está relacionada com a escolha de ferramentas, procedimentos e modelos utilizados para apoiar a implementação de um programa de MPS (o "como").

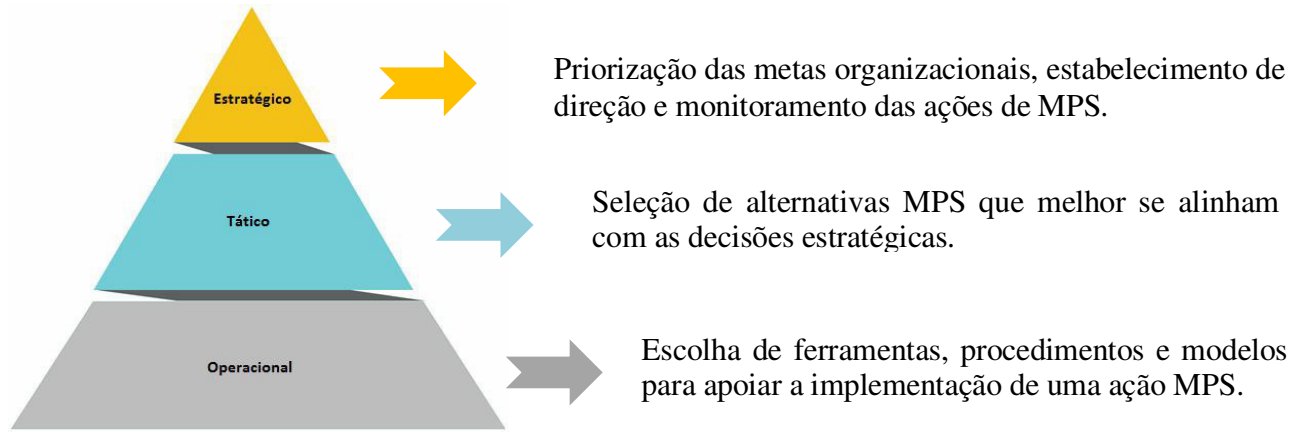

Figura 1. Níveis de tomada de decisão (baseado em Trienekens et al.(2005)). 
O foco do alinhamento estratégico de MPS está na sintonia entre diretores e gestores de nível tático. O nível operacional depende do nível tático pois ambas as decisões são técnicas. $\mathrm{O}$ alinhamento entre metas organizacionais e as decisões táticas implica na tradução da visão empresarial para a visão técnica, que pode ser uma tarefa desafiadora no processo de tomada de decisão de MPS. Esta questão levanta outra conjectura:

$C_{3}$ : $O$ processo de tomada de decisão deve relacionar a priorização dos objetivos organizacionais (decisão estratégica) com a seleção de alternativas de MPS (decisão tática).

Modelos de maturidade para desenvolvimento de software, como CMMI ou MR-MPS-SW, recomendam processos para auxílio na tomada de decisões em projetos de software. O objetivo desses processos é apoiar a tomada de decisões consideradas críticas, por meio de um processo formal que pressupõe critérios estabelecidos para a avaliação de alternativas identificadas. Embora a aplicação desses processos ocorra usualmente em decisões relacionadas a projetos de software, parece aplicável à decisão em iniciativas de MPS. Afinal, "processos de software são também software" (Osterweil 1987), e esforços de melhoria de processos possuem características gerenciais semelhantes a projetos. De fato, a ISO/IEC 33014 (ISO/IEC 2013) recomenda que um programa de melhoria de processo seja implementado como um projeto, com patrocínio definido, gerenciamento de projetos, orçamento, marcos e responsabilidade estabelecidas. Tais aspectos sugerem uma nova conjectura:

C4: A lógica de tomada de decisão deve abranger e registrar critérios aplicados em ambos os níveis de decisões, fornecendo elementos para uma revisão periódica de planos de ação de MPS decorrentes.

Como os problemas de decisão estão diretamente relacionados ao gerenciamento de melhoria de processos, é necessária a revisão de teorias de decisão e de sistemas de apoio à decisão aplicáveis ao alinhamento estratégico de MPS.

\subsection{Teorias de decisão e sistemas de apoio à decisão}

A teoria da decisão é desenvolvida desde meados do século XX com base em contribuições de várias disciplinas, estudadas por economistas, estatísticos, psicólogos, cientistas políticos e sociais ou filósofos. Há dois campos de pesquisa em teoria da decisão: normativo e descritivo (Peterson 2009). O ponto de vista normativo procura produzir prescrições sobre o que decisores devem racionalmente (ou deveriam) fazer em uma decisão. Alternativamente, teorias de decisão descritivas tentam explicar e prever como decisões são realmente tomadas, por meio de processos empíricos decorrentes da psicologia experimental.

As abordagens normativas, foco de nosso estudo, tratam questões sobre como agir quando há incerteza e falta de informação. Exploram também preocupações sobre como um indivíduo pode coordenar suas decisões ao longo do tempo, e de como as pessoas tomam suas decisões em um grupo, envolvendo aspectos sociais e políticos. Gigerenzer e Selten (2001) mencionam que Herbert Simon, ao conceber o termo "racionalidade limitada" (do inglês bounded rationality), usou a metáfora da tesoura, na qual uma lâmina representa as limitações cognitivas dos seres humanos enquanto a outra é a estrutura do ambiente. As limitações e os erros de percepção restringem a 
capacidade dos tomadores de decisão para selecionar a melhor opção a partir de alternativas disponíveis, dificultando assim as decisões ótimas assumidas pelo modelo racional. Pessoas com limitações de tempo, conhecimento e de outros recursos, podem decidir e serem bem-sucedidas em seus ambientes. Assim, estudos devem considerar a necessidade de ambos os lados da tesoura para cortar. O que Simon chama de "ambiente" está relacionado com as necessidades, impulsos ou metas e com a percepção da pessoa (Simon 1956). O autor afirma que as pessoas desenvolvem procedimentos de decisão suscetíveis às restrições, mesmo que esses pareçam insensatos se as restrições forem removidas. No lugar de examinar todas as alternativas possíveis, busca-se apenas encontrar uma solução satisfatória o suficiente em relação a algum critério. Em suma: atinge-se a "satisfação". Este ponto remete a outra conjectura:

C5: Um processo decisório adequado ao alinhamento estratégico de MPS deve equilibrar racionalidade e agilidade, visando ser útil.

A falta de informação adequada leva a uma dependência da intuição, experiência e do julgamento por especialistas (Bhushan e Rai 2004). O problema é abstraído por meio de ponderações, classificações ou julgamento de importância relacionado a um conjunto de atividades, de acordo com seu impacto na situação e com o objetivo das decisões a serem tomadas. Trata-se de um processo de tomada de decisão de múltiplos critérios (do inglês Multiple Criteria Decision Making - MCDM). O Analytic Hierarchy Process (AHP) representa uma abordagem sistemática de MCDM, desenvolvida na década de 1970 por Thomas L. Saaty, para apoiar decisões baseadas na experiência, intuição e em heurísticas. Trata-se de uma metodologia bem definida derivada de princípios matemáticos sólidos (Saaty 2008), que fornece uma abordagem formal a partir da qual uma melhor qualidade das soluções de problemas complexos justifica o tempo investido no processo. Surge assim uma conjectura complementar:

C6: Uma abordagem MCDM (AHP, por exemplo) pode ser útil em um processo de tomada de decisão de MPS.

Bazerman e Moore (2009), adotando uma abordagem descritiva, apresentam limites existentes na tomada de decisões, incluindo a racionalidade limitada, a força de vontade limitada, o interesse limitado, a consciência limitada e a ética limitada. Eles fornecem razões práticas para questionar o uso da intuição na tomada de decisões. Finalmente, o livro fornece seis etapas para melhorar as decisões: (1) usar ferramentas de apoio à decisão, (2) adquirir experiência, (3) evitar viés, (4) analisar racionalmente, (5) obter visão de fora, e (6) identificar vieses em outros. O passo da ferramenta leva ao conceito de Sistemas de Apoio à Decisão (SAD) - do inglês, Decision Support Systems - e seu papel na tomada de decisões. Como a preocupação principal aqui é a compreensão da utilidade percebida, por profissionais, de um processo de tomada de decisão aplicado ao alinhamento estratégico de MPS, o apoio ferramental atua induzindo uma mudança direcionada, como sugere a abordagem de Silver (1990), na qual o SAD assume um papel instrumental com o objetivo de facilitar a coleta de conhecimento sobre o processo de tomada de decisão mais adequado ao contexto. Este conceito reforça outra conjectura:

C7: Uma ferramenta pode melhorar um processo de tomada de decisão de MPS. 


\section{Método de Pesquisa}

O método de pesquisa empregado neste trabalho pode ser descrito por meio do Research Path Schema (RPS) proposto por Stol e Fitzgerald (2015), que sugere a ênfase e ordem dada aos principais elementos presentes em qualquer metodologia de pesquisa. O RPS baseia-se na premissa de que qualquer pesquisa envolva pelo menos três elementos: (a) o conteúdo de interesse, (b) ideias que dão sentido a esse conteúdo, e (c) técnicas ou procedimentos por meio dos quais essas ideias e conteúdos podem ser estudados. Em RPS, esses três elementos são classificados como domínios substantivo, conceitual e metodológico, respectivamente. Dependendo de como esses domínios são combinados, diferentes caminhos podem ser estabelecidos. Neste trabalho, segue-se o caminho observacional dirigido pelo sistema (do inglês system-driven observational path). No caminho observacional o objetivo é coletar observações e explicá-las em termos de um conjunto de conceitos (Stol e Fitzgerald, 2015). O pesquisador define o tópico de interesse (domínio substantivo) e o método de pesquisa.

Conforme mostrado na Figura 2, o foco principal da pesquisa é o conhecimento teórico sobre a utilidade de um processo formal de tomada de decisão para o alinhamento entre as iniciativas de MPS e os objetivos de negócios da organização (domínio substantivo). O metodológico é o domínio secundário. As principais contribuições são observações empíricas (de estudos observacionais iterativos), que levam ao estabelecimento de conceitos (formulação de proposições teóricas) sobre a utilidade de uma solução.

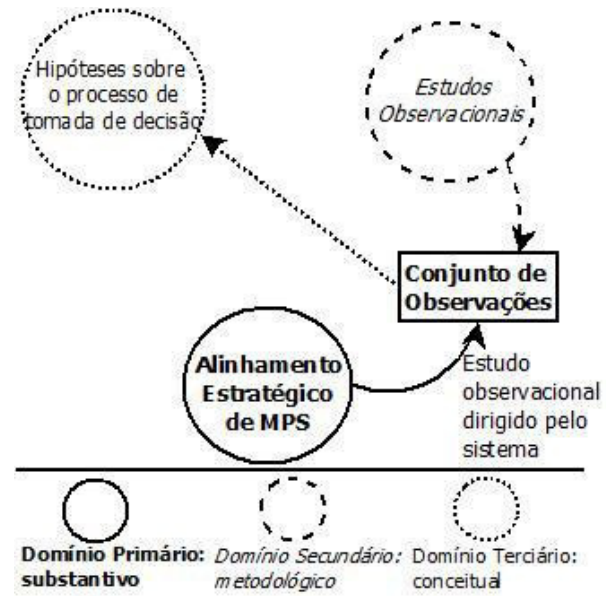

Figura 2. Caminho de pesquisa (baseda em Stol e Fitzgerald (2015)).

A metodologia iterativa proposta por Shull et al. (2001) consiste em três tipos de estudos: viabilidade, observacional e estudo de caso. Associando esta metodologia ao modelo de transferência de tecnologia proposto por Gorschek et al. (2006) e Gorschek (2015) delineou-se um método com três fases de validação: Acadêmica, Estática e Dinâmica. A Validação Acadêmica engloba a elaboração e análise de viabilidade de um processo de tomada de decisão para o alinhamento estratégico de iniciativas de MPS. A fase de Validação Estática engloba uma a avaliação por amostragem objetivamente selecionada de organizações que podem contribuir para a validação estática mencionada por Gorschek (2015). Esta avaliação é realizada por meio de estudos observacionais conforme proposto por Shull et al. (2001). A fase de Validação Dinâmica consiste em estudos de caso em organizações, conduzidos "sem qualquer interferência, ajuda ou 
assistência por parte dos pesquisadores" conforme estabelecido por Gorschek et al. (2006). Este estudo descreve a execução e os resultados obtidos nas duas primeiras fases, que servem de base para a última validação necessária. Embora não baseado no Design Science Research (Wieringa 2014), este trabalho relaciona-se com a teoria do design visto que pretende-se escalar uma tecnologia até a prática. Um processo (como o artefato ou tecnologia a ser escalada para prática) e um SAD (como mecanismo de apoio ao desenho do artefato) definem a solução candidata oferecida.

\subsection{A Validação Acadêmica (Fase 1)}

Na primeira fase os requisitos de um processo de tomada de decisão para o contexto do alinhamento estratégico de MPS são identificados, oferecendo assim uma solução candidata a ser utilizada na fase de Validação Estática. A concepção inicial do processo de apoio à tomada de decisão surge por meio de informações extraídas da literatura e por entrevistas não estruturadas com profissionais decisores em MPS, iniciadas com a pergunta: "Como você define prioridades para iniciativas de MPS e como as alinha aos objetivos organizacionais?”. Uma amostragem intencional de organizações de diferentes portes, mas com certo nível de maturidade do processo de software, fornece informações relevantes sobre técnicas aplicadas pelos decisores para selecionar e priorizar melhorias nos processos. Entrevistas não estruturadas com especialistas no domínio da tomada de decisão também são previstas para o entendimento sobre a inserção de um processo formal e de um SAD em um ambiente de produção.

Para minimizar o viés, três pesquisadores, com diferentes níveis de experiência, analisam os dados da entrevista e elaboram a proposta de solução. A validação da solução candidata em ambiente acadêmico visa a coleta de feedback por pesquisadores experientes e a identificação de falhas que possam ser corrigidas antes da validação por profissionais. Ao todo, cinco autores contribuem para a análise da solução candidata, representando assim, a triangulação por pesquisadores sugerida por Morse (2015).

\subsection{A Validação Estática (Fase 2)}

Como proposto por Gorschek et al. (2006), a Validação Estática envolve a apresentação da solução candidata a ambientes reais com o objetivo de coletar dados para melhorias. O treinamento de profissionais e a execução de casos aplicando o processo de apoio à tomada de decisão visam à coleta de dados, tanto por observações feitas pelos pesquisadores, quanto por entrevistas e questionários realizados junto aos profissionais.

As observações e questionários, triangulados com entrevistas informais e não estruturadas, são o principal método de coleta de dados. Assim como na primeira fase, o objetivo é coletar a visão dos decisores sobre como selecionar melhorias nos processos de software e como alinhar esta iniciativa com as metas organizacionais prioritárias. $\mathrm{O}$ componente ferramental da solução (SAD) atua como um mecanismo (Wieringa 2014b) para auxiliar a obtenção do entendimento sobre o processo de tomada de decisão mais adequado ao alinhamento estratégico de MPS.

A amostragem organizacional objetivamente selecionada visa facilitar o tratamento de questões éticas e o rapport (Guest et al. 2013). Esta amostragem, apresentada na Tabela 1, engloba a diversidade de tamanho, assim como uma variedade de contextos e de maturidade de processo de software, aspecto importante de generalização (Petersen e Wohlin 2009). 
Tabela 1. Amostragem organizacional. Organizações A, B, C e D contribuíram para a Fase 1, enquanto as organizações $D$, E e F contribuíram para a Fase 2.

\begin{tabular}{|c|c|c|c|c|c|c|}
\hline \multirow{2}{*}{ Organização } & Produto & Processos & Práticas & Maturidade & $\begin{array}{c}\text { Estrutura de } \\
\text { decisão }\end{array}$ & $\begin{array}{c}\text { Rigor do } \\
\text { Mercado }\end{array}$ \\
\cline { 2 - 7 } & Segmento & $\begin{array}{c}\text { Definidos ou } \\
\text { Informais }\end{array}$ & $\begin{array}{c}\text { Tradicionais ou } \\
\text { ágeis }\end{array}$ & $\begin{array}{c}\text { Experiência com } \\
\text { modelos MPS }\end{array}$ & $\begin{array}{c}\text { Tamanho da Org.e } \\
\text { número decisores } \\
\text { sobre MPS }\end{array}$ & $\begin{array}{c}\text { Nível de } \\
\text { regulação }\end{array}$ \\
\hline $\mathrm{A}$ & $\begin{array}{c}\text { Sistemas } \\
\text { bancários }\end{array}$ & Definidos & Tradicionais & MR-MPS-SW- C & Grande - 5 & Alto \\
\hline $\mathrm{B}$ & $\begin{array}{c}\text { Gestão } \\
\text { financeira }\end{array}$ & Definidos & Ágeis & MR-MPS-SW- F & Pequeno - 2 & Médio \\
\hline $\mathrm{C}$ & Telecom & Definidos & Tradicionais & CMMI L.3 & Grande -10 & Alto \\
\hline $\mathrm{D}$ & Governo & Definidos & Ágeis & MR-MPS-SW- G & Médio -3 & Médio \\
\hline $\mathrm{E}$ & $\begin{array}{c}\text { Gestão } \\
\text { financeira }\end{array}$ & Informais & Ágeis & Nenhuma & Micro - 1 & Médio \\
\hline $\mathrm{F}$ & ERP & Definidos & Tradicionais & MR-MPS-SW- C & Grande -8 & Baixo \\
\hline
\end{tabular}

Além de observações e de entrevistas informais, um questionário de descrição de contexto (Petersen e Wohlin 2009) e um questionário adaptado do modelo de aceitação de tecnologia (Technology Acceptance Model - TAM) (Davis 1989) avaliam a utilidade percebida e a facilidade de uso da solução em cada caso. O questionário TAM adaptado apresenta quatro questões para análise da utilidade percebida e outras quatro questões para a avaliação da facilidade de uso, abordando os aspectos descritos na Tabela 2.

Tabela 2. Aspectos abordados na questões sobre utilidade e facilidade de uso.

\begin{tabular}{|l|l|}
\hline \multicolumn{1}{|c|}{ Utilidade } & \multicolumn{1}{c|}{ Facilidade de uso } \\
\hline U1: Execução de análise estratégica & F1: Facilidade de aprendizagem \\
\hline U2: Identificação de causas-raiz & F2: Possibilidade de uso sem apoio \\
\hline U3: Ligação de problemas a processos & F3: Entendimento da sequência de passos \\
\hline U4: Avaliação da utilidade percebida & F4: Facilidade de análise estratégica \\
\hline
\end{tabular}

\subsection{A Validação Dinâmica (Fase 3)}

A Validação Dinâmica consiste na entrega da solução a ambientes reais sem que ocorra qualquer interferência por parte dos proponentes da solução. O processo, mesmo útil, pode vir a ser ignorado devido a fatores de confusão (confounding factors) não tratados pelos pesquisadores (Unterkalmsteiner et al. 2012). Além disso, mesmo que os profissionais usem a solução, estes podem não usá-la da maneira prevista (Gorschek, 2015). A Validação Estática coleta as necessidades de treinamento, de documentação, de apoio, e de outros itens que permitirão o uso adequado da solução, ou seja, requisitos para a Validação Dinâmica. Um trabalho futuro, para medir efeitos da solução e como esta é usada na prática, estabelecerá procedimentos de avaliação desta fase.

\section{Resultados e Análise}

O desenvolvimento da solução ocorreu iterativamente pela análise dos resultados de cada fase de validação. As conjecturas iniciais - confirmadas, melhoradas ou alteradas tornaram-se proposições pelo processo de teorização (Recker 2013).

\subsection{Validação Acadêmica}

Foram realizadas entrevistas com seis decisores (D) (das organizações A, B, C e D listadas na Tabela 1) e com três especialistas em tomada de decisão (TD-Esp), sobre a utilidade de um processo formal de tomada de decisão para a seleção e para a priorização de esforços de MPS. 
O processo de tomada de decisão baseado em AHP apoiado por planilhas do Microsoft Excel ${ }^{\circledR}$ foi definido pelos três primeiros autores e avaliado por outros dois pesquisadores (R). Sendo especialistas em Sistemas de Apoio à Decisão e em melhoria de processos de software, esses pesquisadores contribuíram para o refinamento da solução candidata, sendo convidados a participar da análise dos dados da Validação Estática. As sugestões e críticas apresentadas levaram à elaboração de nova versão do processo e à construção de um aplicativo Java, empregados na Validação Estática.

\subsection{Validação Estática}

A coleta de dados sobre a utilidade percebida e facilidade de uso da solução também obteve sugestões de melhorias (tanto para o processo quanto para o software), por meio de respostas às questões abertas inseridas ao final do questionário adaptado do TAM. $\mathrm{O}$ questionário empregado utilizou uma escala de Likert de seis pontos, omitindo o valor médio por não fornecer informações sobre a opinião do participante, ou seja, sobre concordar ou discordar (Laitenberger e Dreyer 1998). Foi atribuída uma faixa de valores numéricos entre 1 e 3 para cada tipo de resposta. As respostas positivas receberam valores de 1 a 3 (concordo parcialmente, concordo e concordo fortemente). As respostas negativas receberam valores de -1 a -3 (discordo parcialmente, discordo e discordo fortemente). Com um total de 12 questionários aplicados (abrangendo as organizações D, E e F) poderia ser obtido um valor máximo de 36 no somatório ( $\Sigma$ ) de cada item. Os resultados obtidos são apresentados na Tabela 3:

Tabela 3. Resultados das respostas ao questionário.

\begin{tabular}{|c|c|c|c|c|c|c|c|c|}
\cline { 2 - 9 } \multicolumn{1}{c|}{} & F1 & F2 & F3 & F4 & U1 & U2 & U3 & U4 \\
\hline $\boldsymbol{\Sigma}$ & 25 & $\mathbf{2 1}$ & 32 & 29 & 32 & 25 & 26 & 29 \\
\hline$\overline{\mathbf{X}}$ & 2,08 & $\mathbf{1 , 7 5}$ & 2,67 & 2,42 & 2,67 & 2,08 & 2,17 & 2,42 \\
\hline $\boldsymbol{\sigma}$ & 0,29 & $\mathbf{0 , 4 5}$ & 0,65 & 0,67 & 0,49 & $\mathbf{0 , 6 7}$ & 0,58 & $\mathbf{0 , 6 7}$ \\
\hline
\end{tabular}

Pode-se perceber que a média obtida nos itens indica concordância de que a solução proposta é útil e fácil de usar. No entanto, duas preocupações surgiram da análise deste resultado. Primeiro, o item F2 (Possibilidade de uso sem apoio) apresenta um baixo valor de média de concordância, associado a baixo desvio padrão. Isto indica que o processo demanda apoio para seu uso efetivo. Além disso, os seguintes comentários contribuíram para a análise desta questão:

"A análise da causa-raiz só será eficaz com o apoio de consultoria." (D2-org D) "É difícil estabelecer os critérios de decisão sem apoio de especialistas." (D1-org D) "Tivemos dificuldades em definir a granularidade dos problemas, gerando assim muitas alternativas para serem avaliadas." (D2-org F)

O feedback acima demonstra a necessidade de melhor apoio de treinamento para a transferência adequada da solução para a prática (tutoriais em vídeo, por exemplo). No entanto, pode ser recomendável ainda o eventual apoio de consultoria, pelo menos no primeiro uso.

A segunda preocupação diz respeito a dados oriundos de observações. Foi observado certo desconforto e impaciência, por parte de um decisor, durante a execução do processo. De fato, ao triangular esta observação com dados do questionário, este decisor é o único a atribuir valores baixos nas questões relacionadas à utilidade percebida, contribuindo assim para o alto desvio padrão resultante nos quesitos $\mathrm{U}_{2}$ e $\mathrm{U}_{4}$. 
O seguinte extrato de repostas ao questionário corroboram a percepção: "Senti falta de gestão de mudanças nas decisões." (D1-org F)

Este feedback motivou a triangulação com uma entrevista complementar realizada com o decisor 2 da mesma organização. $O$ seguinte dado surge quando perguntada a opinião sobre o problema de mudanças nas decisões tomadas:

"Infelizmente, o processo de decisão depende do perfil do decisor e da cultura organizacional. Em nossa organização, mesmo as decisões estratégicas são voláteis [sic]...” (D2-org F)

Devido à posição de liderança do informante (D1 indica ser o decisor com mais alto nível hierárquico na organização), foi realizada nova triangulação com os seguintes dados de entrevista com especialista em tomada de decisão:

"Meu amigo... (mostrando desânimo em sua voz) alguns gerentes evitam processos formais de tomada de decisão devido a motivos não publicáveis. Tais motivações podem ser políticas, sociais ou mesmo antiéticas..." (TD-Esp1)

Consequentemente, devido ao risco de insucesso na efetiva transferência para a prática na presença de cenários envolvendo decisores de nível estratégico com perfil similar, postulou-se nova proposição $\mathrm{P}_{8}$. Os dados coletados confirmaram as conjecturas $\mathrm{C}_{1}, \mathrm{C}_{4}$ e $\mathrm{C}_{5}$. Além da nova proposição $\mathrm{P}_{8}$, foi alterada $\mathrm{C}_{2}$ e aprimoradas $\mathrm{C}_{3}, \mathrm{C}_{6}$ e $\mathrm{C}_{7}$. $\mathrm{A}$ Tabela 4 descreve as proposições resultantes $\left(\mathrm{P}_{1}\right.$ a $\left.\mathrm{P}_{8}\right)$.

Tabela 4. Proposições decorrentes.

\begin{tabular}{|c|c|}
\hline $\mathbf{P}_{\mathbf{n}}$ & Proposições \\
\hline$P_{1}$ & $\begin{array}{l}\text { O alinhamento estratégico de MPS envolve o planejamento e o monitoramento de atividades, } \\
\text { demandando tanto sistemas para apoio à tomada de decisão quanto para a mensuração de } \\
\text { resultados. }\end{array}$ \\
\hline$P_{2}$ & $\begin{array}{l}\text { O alinhamento estratégico de MPS envolve dois papéis dutas partes interessadas principais: } \\
\text { decisores dos níveis estratégico e tático. diretores e gerentes de MPS. } \\
\text { "Concordo com os dois níveis de decisão, mas na minha empresa eu tomo as duas decisões às } \\
\text { vezes." (D1-org B) }\end{array}$ \\
\hline$P_{3}$ & $\begin{array}{l}\text { O processo de tomada de decisão deve relacionar a priorização dos objetivos organizacionais } \\
\text { (decisão estratégica) com a seleção de alternativas de MPS (decisão tática). } \\
\text { Aprimoramento: Critérios de decisão devem ser estabelecidos pelo nivel estratégico, mas } \\
\text { alternativas de MPS devem ser identificadas antes da seleção dos critérios de decisão. } \\
\text { "A determinação de critérios para a seleção de alternativas normalmente depende das opçães } \\
\text { escolhidas. O nível tático deve determinar alternativas viáveis para que possam ser estabelecidos } \\
\text { os critérios de seleção." (TD-Esp2) }\end{array}$ \\
\hline$P_{4}$ & $\begin{array}{l}\text { A lógica de tomada de decisão deve abranger e registrar critérios aplicados em ambos os níveis de } \\
\text { decisões, fornecendo elementos para uma revisão periódica de planos de ação de MPS } \\
\text { decorrentes. }\end{array}$ \\
\hline$P_{5}$ & $\begin{array}{l}\text { Um processo decisório adequado ao alinhamento estratégico de MPS deve equilibrar } \\
\text { racionalidade e agilidade, visando ser útil. }\end{array}$ \\
\hline$P_{6}$ & 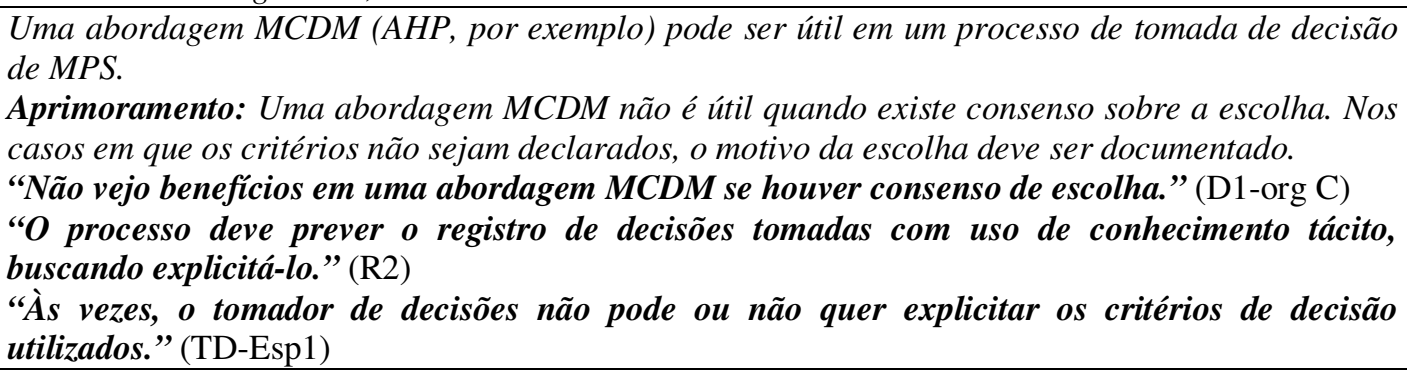 \\
\hline
\end{tabular}




\begin{tabular}{|c|l|}
\hline$P_{7}$ & $\begin{array}{l}\text { Uma ferramenta pode melhorar um processo de tomada de decisão de MPS. } \\
\text { Aprimoramento: A usabilidade, o controle de acesso e o controle de versões de decisões são } \\
\text { requisitos relevantes de uma ferramenta útil ao processo de tomada de decisão. } \\
\text { "O uso de planilhas pode causar avaliação negativa pelos usuários ...” (R1) } \\
\text { "Acho fundamental o acesso controlado aos registros de decisões tomadas, seja para revisão } \\
\text { futura ou para comparação em caso de problemas semelhantes.”(D1-org A) }\end{array}$ \\
\hline$P_{8}$ & $\begin{array}{l}\text { O sucesso de um processo formal de apoio à decisão para o alinhamento estratégico de iniciativas } \\
\text { de MPS depende diretamente do grau de envolvimento e comprometimento da alta direção da } \\
\text { organização (decisores do nível estratégico). }\end{array}$ \\
\hline
\end{tabular}

Em outras palavras, não é possível adotar um processo que envolve a participação direta da alta gerência, sem seu compromisso claro e definido. Humphey (1989), em seu primeiro princípio básico de mudança de processo, estabelece que "grandes mudanças no processo de software devem começar no topo".

A seguir, é descrito o componente do processo da solução. A ferramenta deverá ser aprimorada e por este motivo não é apresentada como resultado do presente trabalho.

\subsection{A Solução proposta}

Devido à limitação de espaço, não é viável detalhar os procedimentos envolvidos em cada atividade do processo ilustrado na Figura 3. O objetivo é fornecer sua visão geral.

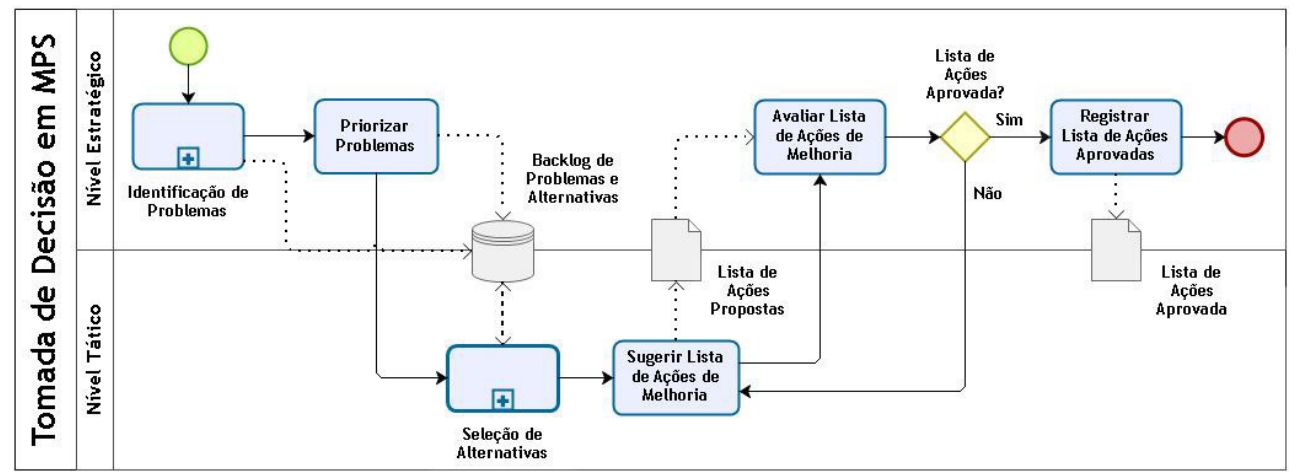

Figura 3. O processo de apoio à tomada de decisão.

Os papéis relacionados aos decisores nos níveis estratégico e tático podem ser combinados em uma única pessoa, como ocorreu na organização E (ver Tabela 1). Uma ferramenta (SAD) armazena informações relevantes sobre as escolhas entre alternativas e critérios adotados. Em alguns casos, o SAD apoiará decisões por meio de dados históricos. O primeiro passo do processo, representado por um subprocesso, possui duas atividades mostradas na Figura 4.

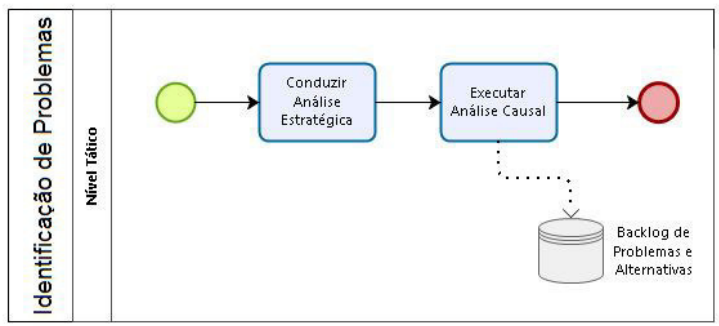

Figura 4. 0 subprocesso Identificação de Problemas. 
Para identificar problemas, a análise de forças, fraquezas, oportunidades e ameaças, ou Análise SWOT (Pickton e Wright 1998), extrai problemas no nível organizacional. Os problemas são analisados quanto a possíveis causas-raiz, usando os cinco porquês (Myszewski 2013) ou outro método de análise causal. Os problemas considerados causas-raiz são priorizados usando uma matriz Gravidade-UrgênciaTendência (GUT) resultando na lista ordenada de problemas (Backlog de problemas).

Seleção de Alternativas é outro subprocesso, detalhado na Figura 5. Como mencionado anteriormente, a versão aprimorada da solução oferece um caminho ágil para registrar uma escolha consensual (vide proposição $P_{5}$ ). As alternativas devem ser identificadas antes da seleção dos critérios de decisão (vide proposição $\mathrm{P}_{3}$ ).

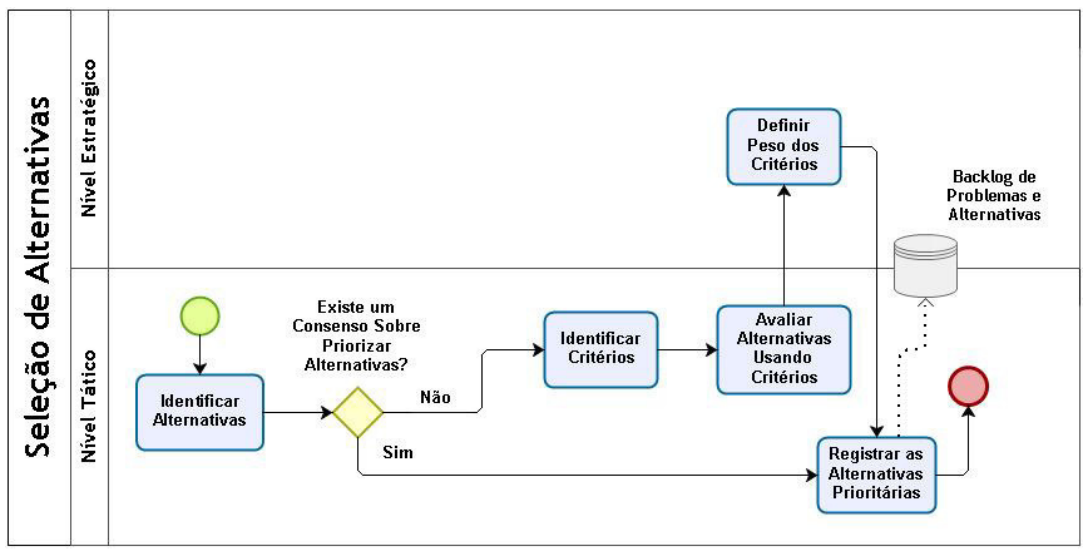

Figura 5.0 subprocesso Seleção de Alternativas.

As alternativas selecionadas compõem uma lista que é submetida à aprovação do nível de decisão estratégico, garantindo assim o alinhamento das ações de MPS com os objetivos da alta direção.

\section{Ameaças à Validade}

Apesar de esforços para reduzir ameaças à validade interna, selecionando uma amostra diversificada de organizações e empregando a triangulação em diferentes níveis (Morse 2015), este estudo pode apresentar viés devido à amostragem intencional. O objetivo foi facilitar o tratamento de questões éticas e o rapport. No entanto, esse rapport pode ser responsável por outra ameaça à validade, pois a parceria entre os pesquisadores e as instituições colaboradoras pode ter provocado a presença de um efeito Hawthorne (McCambridge et al. 2014), induzindo respostas com viés positivo.

O contexto é importante para a generalização (Petersen e Wohlin 2009). Assim, falhas em sua definição podem representar uma ameaça à validade externa. A amostragem intencional para a Validação Estática pode representar uma ameaça à validade externa da investigação, uma vez não ser possível generalizar resultados com base em apenas três casos. No entanto, em harmonia com a afirmação de Corbin e Strauss (2015) de que "a generalização não é o objetivo da pesquisa qualitativa", buscou-se obter credibilidade no estudo por meio de outros procedimentos. Como recomendado por Creswell e Miller (2000), com a aplicação de diferentes tipos de triangulação (de pesquisadores, de métodos e de dados), peer review, debriefing e pelo trabalho em estreita colaboração com os participantes, acredita-se ter alcançado a confiabilidade. 


\section{Conclusões e Trabalhos Futuros}

A principal contribuição deste estudo foi a ampliação do conhecimento sobre o processo de tomada de decisão aplicado ao alinhamento entre as iniciativas de melhoria de processos de software e metas organizacionais. As suposições iniciais foram aprimoradas no transcorrer da pesquisa tornando possível obter confiança suficiente para a sequência do trabalho futuro (Fase 3).

O aprimoramento conceitual sobre a tomada de decisões no alinhamento estratégico de MPS confirma que o processo é útil e necessário para a sintonia entre decisões estratégicas e táticas e, consequentemente, para o sucesso das ações planejadas pelo nível tático (gestores de MPS). A fase de Validação Dinâmica determinará se a solução oferecida poderá ser adequadamente transferida para a indústria. Igualmente importante, um processo para o monitoramento e gestão de planos de ação derivados das decisões, deverá ser concebido e validado.

Por fim, o estudo desperta a necessidade de investigação sobre os fatores, relacionados à capacidade cognitiva humana e interação social, que implicam em comportamentos dos decisores e refletem nos processos de apoio à tomada de decisões. Tal conclusão está diretamente relacionada ao estudo de Lenberg et al. (2015).

\section{Referências}

Bayona, Sussy, Jose a. Calvo-Manzano, e Tomás San Feliu. 2012. "Critical Success Factors in Software Process Improvement: A Systematic Review." Software Process Improvement and Capability Determination, 1-12.

Bazerman, Max H, e Don A Moore. 2009. Judgment in Managerial Decision Making. 7 th ed. New Jersey: John Wiley \& Sons, Inc.

Bhushan, Navneet, e Kanwal Rai. 2004. Strategic Decision Making: Applying the Analytical Hierarchy Process. 1st ed. London: Springer-Verlag.

Corbin, Juliet, e Anselm Strauss. 2015. Basics of Qualitative Research: Techniques and Procedures for Developing Grounded Theory. London: SAGE Publications.

Creswell, John W., e Dana L. Miller. 2000. "Determining Validity in Qualitative Inquiry." Theory Into Practice 39 (3): 124-30.

Davis, Fred D. 1989. "Perceived Usefulness, Perceived Ease of Use, and User Acceptance of Information Technology." MIS Quarterly 13 (3): 319.

Dybå, Tore. 2005. “An Empirical Investigation of the Key Factors for Success in Software Process Improvement." IEEE Transactions on Software Engineering 31 (5): 410-24.

Ebert, Christof. 1999. "Technical Controlling and Software Process Improvement." Journal of Systems and Software 46 (1): 25-39.

Gigerenzer, Gerd., e Reinhard. Selten. 2001. Bounded Rationality: The Adaptive Toolbox. MIT Press.

Gorschek, Tony. 2015. "How to Increase the Likelihood of Successful Transfer to Industry -- Going Beyond the Empirical." In 2015 IEEE/ACM 3rd International Workshop on Conducting Empirical Studies in Industry, 10-11. IEEE. 
Gorschek, Tony, Per Garre, Stig Larsson, e Claes Wohlin. 2006. "A Model for Technology Transfer in Practice." IEEE Software 23 (6): 88-95.

Guest, Greg, Emily E. Namey, e Marilyn L. Mitchell. 2013. Collecting Qualitative Data: A Field Manual for Applied Research. London: SAGE Publications Ltd.

Hastie, Reid., e Robyn M. Dawes. 2010. Rational Choice in an Uncertain World: The Psychology of Judgment and Decision Making. London: SAGE Publications Ltd.

Humphrey, Watts S. 1989. Managing the Software Process. Boston, MA, USA: Addison-Wesley Longman Publishing.

ISACA. 2012. COBIT 5: A Business Framework for the Governance and Management of Enterprise IT. ISACA.

ISO/IEC. 2013. "ISO/IEC TR 33014:2013: Information Technology - Process Assessment - Guide for Process Improvement." International Organization for Standardization.

- 2015. "ISO/IEC 38500:2015 - Information Technology - Governance of IT for the Organization." International Organization for Standardization.

Johnson, Phil. 2004. "Analytic Induction." In Essential Guide to Qualitative Methods in Organizational Research, 165-79. London: SAGE Publications Ltd.

Laitenberger, O., e H.M. Dreyer. 1998. "Evaluating the Usefulness and the Ease of Use of a Web-Based Inspection Data Collection Tool." In Proceedings Fifth International Software Metrics Symposium. Metrics, 122-32. IEEE Comput. Soc.

Lenberg, Per, Robert Feldt, e Lars Göran Wallgren. 2015. "Behavioral Software Engineering: A Definition and Systematic Literature Review." Journal of Systems and Software 107 (September): 15-37.

Lepmets, Marion, e Tom McBride. 2012. "Process Improvement for the Small and Agile.” In EuroSPI 2012, 310-18. Springer, Berlin, Heidelberg.

Lepmets, Marion, Tom McBride, e Eric Ras. 2012. "Goal Alignment in Process Improvement." Journal of Systems and Software 85 (6). Elsevier Inc.: 1440-52.

McCambridge, Jim, John Witton, e Diana R. Elbourne. 2014. "Systematic Review of the Hawthorne Effect: New Concepts Are Needed to Study Research Participation Effects." Journal of Clinical Epidemiology 67 (3): 267-77.

Merriam, Sharan B. 2009. Qualitative Research: A Guide to Design and Implementation. San Francisco: Jossey-Bass.

Morse, Janice M. 2015. "Critical Analysis of Strategies for Determining Rigor in Qualitative Inquiry." Qualitative Health Research 25 (9). SAGE PublicationsSage CA: Los Angeles, CA: 1212-22.

Münch, Jürgen, Ove Armbrust, Martin Kowalczyk, e Martín Soto. 2012. "Process Improvement." In Software Process Definition and Management, 139-76.

Myszewski, Jan M. 2013. “On Improvement Story by 5 Whys.” Edited by Madhav Sinha. The TQM Journal 25 (4). Emerald Group Publishing Limited: 371-83. 
Osterweil, Leon J. 1987. "Software Processes Are Software Too." In Proceedings of the 9th International Conference on Software Engineering, 2-13.

Petersen, Kai, e Claes Wohlin. 2009. "Context in Industrial Software Engineering Research." In Proceedings of the 2009 3rd International Symposium on Empirical Software Engineering and Measurement, 401-4. IEEE Computer Society.

Peterson, Martin. 2009. An Introduction to Decision Theory. Cambridge: Cambridge University Press.

Pickton, David W., e Sheila Wright. 1998. "What's Swot in Strategic Analysis?" Strategic Change 7 (2): 101-9.

Qumer, A., e B. Henderson-Sellers. 2008. "A Framework to Support the Evaluation, Adoption and Improvement of Agile Methods in Practice." Journal of Systems and Software 81 (11): 1899-1919.

Recker, Jan. 2013. Scientific Research in Information Systems. Berlin: Springer Berlin Heidelberg.

Ringstad, Mats Angermo, Torgeir Dingsøyr, e Nils Brede Moe. 2011. "Agile Process Improvement: Diagnosis and Planning to Improve Teamwork." In , 167-78. Springer, Berlin, Heidelberg.

Saaty, Thomas L. 2008. "Decision Making with the Analytic Hierarchy Process." International Journal of Services Sciences 1 (1): 83.

Shull, Forrest, Jeffrey Carver, e Guilherme H. Travassos. 2001. "An Empirical Methodology for Introducing Software Processes." ACM SIGSOFT Software Engineering Notes 26 (5): 288.

Silver, Mark S. 1990. "Decision Support Systems: Directed and Nondirected Change." Information Systems Research 1 (1). INFORMS: 47-70.

Simon, Herbert A. 1956. "Rational Choice and the Structure of the Environment." Psychological Review 63 (2). American Psychological Association: 129-38.

Stol, Klaas-Jan, e Brian Fitzgerald. 2015. "Theory-Oriented Software Engineering." Science of Computer Programming 101 (April): 79-98.

Trienekens, Jos J.M., Rob J Kusters, Ben Rendering, e Kees Stokla. 2005. "BusinessOriented Process Improvement: Practices and Experiences at Thales Naval The Netherlands (TNNL)." Information and Software Technology 47 (2): 67-79.

Unterkalmsteiner, Michael, Tony Gorschek, A. K. M. M. Islam, Chow Kian Cheng, Rahadian. B. Permadi, e Robert Feldt. 2012. "Evaluation and Measurement of Software Process Improvement - A Systematic Literature Review." IEEE Transactions on Software Engineering 38 (2): 398-424.

Vasconcellos, F.J.S., G.B. Landre, J.A.O.G. Cunha, J.L. Oliveira, R.A. Ferreira, e A.M.R. Vincenzi. 2017. "Approaches to Strategic Alignment of Software Process Improvement: A Systematic Literature Review." Journal of Systems and Software 123: 45-63.

Wieringa, Roel. 2014. Design Science Methodology for Information Systems and Software Engineering. Berlin, Heidelberg: Springer Berlin Heidelberg. 